\title{
Prognostics and Health Monitoring for Lithium-ion Battery
}

\author{
Yinjiao Xing \\ Center for Prognostics and System Health Management \\ City University of Hong Kong, \\ Hong Kong \\ K.-L. Tsui \\ Dept. of Manufacturing Engineering and Engineering \\ Management \\ City University of Hong Kong \\ Hong Kong
}

\author{
Qiang Miao \\ Center for Advanced Life Cycle Engineering (CALCE) \\ University of Maryland, College Park \\ Maryland, USA \\ Michael Pecht \\ Center for Advanced Life Cycle Engineering (CALCE) \\ University of Maryland, College Park \\ Maryland, USA \\ Email: mgpecht@cityu.edu.hk
}

\begin{abstract}
Health monitoring is used to analyze and predict the battery health status. However, no matter what health monitoring methods and parameters are, a major aim is to improve the battery reliability through surveillance and prognostics. Hence, the latest known methods of state estimation and life prediction based on battery health monitoring are discussed in this paper. Through comparing their characteristics respectively, a prognostics-based fusion technique is proposed that combines physics-of-failure (PoF) with data-driven technology. The fusion approach not only investigates battery failure mechanism caused by environmental and internal characteristics, but also assesses parameters with aid of real-time health monitoring. The specific method is presented to realize the estimation on remaining useful life (RUL) of batteries.
\end{abstract}

Keywords-Prognostics; Health Monitoring; Lithium-ion Battery; Fusion technique; RUL; PoF;

\section{INTRODUCTION}

Lithium-ion battery has been widely applied from hand-held electronics to electric vehicles by virtue of its comprehensive performance. As a critical component of power supplies, battery reliability has a primary effect on an overall system. Battery failures cause performance degradation, potential safety hazard, and even catastrophic result (e.g., the laptop recall event of Dell in 2006 [1] and HP in 2009 [2]). Therefore, accurate state-of-charge (SOC), state-of-health $(\mathrm{SOH})$ and remaining useful life (RUL) of a battery are significant to both manufacturers and end users.

Many methodologies have been used to conduct state estimation and life prediction of batteries. Each of them falls into two main categories: physics-of-failure (PoF), and data-driven techniques. PoF is used to determine the reliability of batteries in terms of RUL under a specific failure mechanism due to a specific load condition. However, it cannot identify the correlated mechanisms and faults. The data-driven techniques are able to detect the fault, but they cannot distinguish between different failure mechanisms. Thus, we propose a fusion technique that combines PoF with data-driven method on the reliability of the battery. Data-driven approaches contribute to distinguish the parameters relevant to the observed degradation and choose the PoF models correspondingly. Under the accelerated life test, RUL can be determined for a specific failure mechanism under certain load. The proposed method not only gives us a more reasonable and accurate prediction but also improves the cost efficiency during a battery qualification testing.

\section{CORE ISSUES IN BATtery HEALTH MONTTORING}

\section{A. Monitored Parameters}

Health monitoring for Lithium-ion battery is conducted to combine fundamental understanding of degradation performance and common monitoring methods. With the development of telecommunication techniques, more parameters have been monitored to form a further understanding of battery degradation.

TABLE I. BATtery MONitoered Parameters [3]

\begin{tabular}{|c|c|c|}
\hline Categories & Objectives & SOC/SOH \\
\hline $\begin{array}{c}\text { Voltage (Cut-off, } \\
\text { High/low) }\end{array}$ & Indicate rectifier & $\begin{array}{c}\text { Indicate state of charge, cannot } \\
\text { predict capacity }\end{array}$ \\
\hline $\begin{array}{c}\text { Battery } \\
\text { Conductance }\end{array}$ & Find weak cells & $\begin{array}{c}\text { No need for battery discharge to } \\
\text { indicate relative state of health }\end{array}$ \\
\hline $\begin{array}{c}\text { Temperature / } \\
\text { Temperature } \\
\text { Difference }\end{array}$ & $\begin{array}{c}\text { Show high } \\
\text { battery } \\
\text { temperature }\end{array}$ & $\begin{array}{c}\text { Combine with other data to } \\
\text { verify battery fault }\end{array}$ \\
\hline Battery Discharge & $\begin{array}{c}\text { Indicate } \\
\text { discharge is in } \\
\text { progress }\end{array}$ & $\begin{array}{c}\text { Capacity prediction is based on } \\
\text { history. Only reports what has } \\
\text { happened }\end{array}$ \\
\hline Run Time & $\begin{array}{c}\text { Require battery } \\
\text { discharge and } \\
\text { history }\end{array}$ & $\begin{array}{c}\text { Complex \& variable calculations } \\
\text { needing time, temperature, DC } \\
\text { current, and history }\end{array}$ \\
\hline $\begin{array}{c}\text { Offline Battery } \\
\text { Alarm }\end{array}$ & $\begin{array}{c}\text { Detects battery } \\
\text { availability }\end{array}$ & $\begin{array}{c}\text { No capacity prediction (indicates } \\
\text { battery is connect to system) }\end{array}$ \\
\hline
\end{tabular}


Table I shows relevant monitored parameters to battery health in the current battery industry. The objectives of health monitoring are to detect and predict the remaining charge and capacity of batteries, which will provide meaningful and accurate indication to the battery management.

\section{B. Health Monitoring}

Usually, SOC, SOH and RUL of batteries are used to characterize the battery health status. Through monitoring parameters mentioned above, these attributes can be estimated accurately. SOC is defined as a ratio of the remaining capacity and the rated capacity [4]. SOH is used to describe the physical condition of the battery, which is commonly characterized by the loss of rated capacity as shown in (2). $Q_{\max }$ is the maximal releasable capacity, which is equal to $Q_{\text {rated }}$ for a brand-new battery. However, it declines as a function of time through consuming and aging.

$$
\begin{gathered}
S_{O C}=\frac{Q_{\text {present }}}{Q_{\max }} \times 100 \% \\
S_{O H}=\frac{Q_{\text {max }}}{Q_{\text {rated }}} \times 100 \%
\end{gathered}
$$

In the battery manufacturing industry, State-of-Life (SOL) is introduced to quantify the RUL of battery as the maximal capacity falling below $80 \%$ of its initial rated capacity [5]. SOL is a prognostic metric. The number of charge-discharge cycles related to the specific performance (i.e. $80 \%$ of the nominal capacity) is the battery cycle life. In this paper, degradation trend of the time-varying capacity is tracked, and the time-to-failure is estimated to realize the proposed approach.

\section{HEALTH MONITORING BASED ON FAILURE MECHANISMS}

\section{A. Failure Mechanisms}

Batteries are electrochemical devices, which convert between chemical energy and electrical energy by means of controlled chemical reactions between a set of active chemicals. Unfortunately, there is a slow deterioration of the chemicals as a result of unavoidable, unwanted chemical actions. For example, the crystal or dendrite growth changes the morphology of particles or causes the loss of electrolyte. These are the inherent failure mechanisms but barely perceptible to the users even to some manufacturers. Thus, most manufacturers test on the observable physical characteristics, which can be reflected due to the internal mechanisms. The common monitored parameters include:

Discharge rate - the higher the discharge rate, the lower the discharged capacity [6].

Depth of Discharge (DOD) - cycling at a reduced depth of discharge improves the cycle life of a battery, decreases capacity fading, and slows down the changes observed in the shape of the discharge curves in Guena's paper [7].

Self-discharge/recharge - a main failure cause due to the loss of capacity occurring at the negative electrode. It is restricted by the electronic conductivity of the Solid-Electrolyte-Interface (SEI) layer [8]. Some fraction of voltage and capacity will increase at the next cycle during rest, and called self-recharge process.

Temperature - high temperature reduces internal resistance and causes high self-discharge. The high temperature situation increases battery's performance temporarily, however, it makes the degradation of lithium-ion batteries much faster than that at the normal situation [9].

Besides, other physical causes, such as high voltage, abuse and mechanical stresses, will also lead to failure. However, these causes can be avoided by protection circuit or assured to work in a reasonable range through setting alarm signals. Hence, these causes are neglected in our current study.

\section{B. Health Monitoring Methods}

Specific Gravity Measurement is a traditional way of determining the charge condition of lead acid batteries. However, it is not suitable for other cell chemistries, such as lithium-ion battery.

Direct measurement of the capacity can be realized at a constant discharge current rate because the charge is equal to the current multiplies the time. However, the discharge current varies continuously in a nonlinear way. Moreover, this method needs to know how much charge the battery contained before discharging. Thus, the manufacturers cannot achieve the initial charge and capacity information of the battery during qualification testing.

Coulomb counting [10] measures from the charge transferred in to out of the cell by accumulating the current with time. A fully charged situation is viewed as the calibration reference point. However, as the battery performance fading, the reference point should be compensated continuously for the loss of the capacity.

As a black box, an inherent failure of battery caused by internal parameters is more expected to be identified. With the development of the measurement technology and reduced cost, Electrochemical Impedance Spectroscopy (EIS), a non-invasive method, has been used to characterize the battery capacity degradation through variations of the internal parameters. In other words, battery life can be determined and predicted from the impedance changes. EIS is a significant technology in the construction of "PoF mechanism" including coatings, anodized films, corrosion inhibitors and detailed information of the system under testing [11]. By injecting a small AC signal into a battery, the responses will be extracted from the equivalent circuit model (fig.1). The corresponding Nyquist plot (fig.2) can be used to explore the equivalent impedance parameters, which generally include double layer capacitor $\mathrm{C}_{\mathrm{DL}}$, the charge transfer resistance $\mathrm{R}_{\mathrm{CT}}$, the Warburg impedance $R_{W}$, and the electrolyte resistance $R_{E}$.

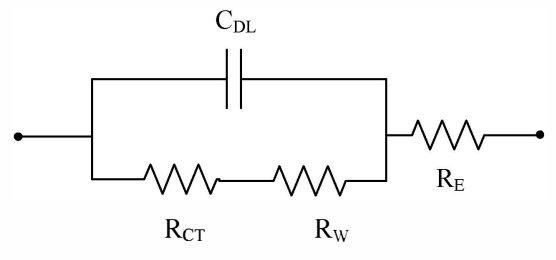

FIg.1 EQUiVALENT LUMPED BATTERY MODEL (RANDLES MODEL)

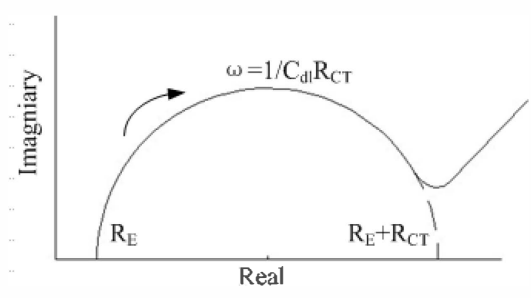

FIg.2 NYQUist Plot FOR EQUIVALENT RANDLES CIRCUIT 


\section{Modeling, State Estimation, AND Life PRediction}

\section{A. Existing Modeling}

Different models, such as analytical models and degradation models, have been developed to analyze the battery status.

Rakhmatov [12] developed an analytical model for generic batteries. It is able to predict the time-to-failure for a given load. The model was developed from the Peukert Equations: $C=I^{\beta} T$ where $C$ denotes the theoretical capacity of a battery, and $\beta$ is an appropriate coefficient [13] that needs to be estimated for analytical model. $I$ and $T$ are the average load current and time respectively during discharging process. To present the degradation trend, the capacity can be delineated as a function of current with time-varying through tracking the concentration of species.

$$
C(t)=l(t)+r(t)
$$

Where $l(t)=\int_{0}^{t} i(\tau) d \tau, r(t)=2 \sum_{0}^{+\infty} \int_{0}^{r} i(\tau) \cdot e^{-m^{2} \gamma^{2}(T-\tau)} d \tau$

$$
C \approx \sum_{k=1}^{N} I_{k-1}\left(t_{k}-t_{k-1}+2 \sum_{m=1}^{\infty} \frac{e^{-\gamma^{2} m^{2}\left(T-t_{k}\right)}-e^{-\gamma^{2} m^{2}\left(T-t_{k-1}\right)}}{\gamma^{2} m^{2}}\right)
$$

Where $C(t)$ is viewed as the lost capacity of the battery under the specific $i(t), l(t)$ is the capacity consumed by external circuit, and $r(t)$ is the remaining unavailable charge during discharging. (4) is the numerical expression of (3) in the discrete current expression while (5) is a modified expression of Peukert's law with general case.

$$
C \approx\left[\frac{\sum_{k=1}^{N} I_{k-1}\left(t_{k}-t_{k-1}\right)}{T}\right]^{\beta} T
$$

To predict the lifetime $T$, the parameter pair $(C, \beta)$ should be estimated from the experimental data for the modeled battery by virtue of fitting the load values for a given set of observed lifetimes.

However, the analytical model employs an equivalent expression as (6) and goes through a high level of abstraction of the battery electrochemical phenomena. That means it cannot provide other battery physical features in terms of internal resistance and open-circuit voltage.

Thomas utilized a simple empirical degradation model taking into account temperature factor from the statistical methodology [14]. The error model provides a basis for assessing a level of measurement error based on the experiment data.

$$
\mu(X ; t)=1+\exp \left\{\alpha_{0}+\alpha_{1} \cdot \frac{1}{T}\right\} \cdot t^{m}
$$

Where $\mu(X ; t)$ denotes the mean of relative resistance (relative to the initial resistance).

Take the log formation of (6):

$$
\log (\mu(T ; t)-1)=\alpha_{0}+\alpha_{1} \cdot \frac{1}{T}+m \cdot \log t
$$

(7) is used to fit the degradation model through robust linear regression.

$$
\zeta_{i}(X ; t)=\delta_{i} \cdot(\mu(X ; t)-1)+\pi_{i}(t)
$$

While $\zeta_{i}(X ; t)$ is the error including two parts: a unique effect to each cell and measurement error. Thus, the overall model parameters that need to be estimated include: $\alpha_{0}, \alpha_{1}$, $m, \sigma_{\delta}^{2}, \sigma_{\pi}^{2}$. The latter two represent the variances of $\delta_{i}$ and $\pi_{i}$, respectively. The degradation model was fitted under $40^{\circ} \mathrm{C}, 47.5^{\circ} \mathrm{C}$, and $55^{\circ} \mathrm{C}$. The $30^{\circ} \mathrm{C}$ data were used to validate the model.

This degradation model is an empirical-based model as a function of temperature and time to predict RUL. The key issue is whether different temperature level will take a risk of the internal chemical reactions of battery. Thus, the critical temperature should be used to distinguish between different models. If temperature does not give rise to other reactions in a certain range, the predictive result will be more convincing. Except for temperature, many other parameters leading to the degradation are ignored in the empirical model. Thus, we need to adopt PoF methods to quantify and distinguish the distinctive level caused by a specific factor.

EIS technology has been developed to find the linear correlation between a battery capacity and internal impedance [15]. The internal resistance is able to characterize the battery degradation. Through comparing different regression models, Gebel finalized relevance vector machine (RVM) to fit the representative aging curves in terms of EIS data. RVM is used for model development by using a kernel-based regression/classification similar to support vector machine (SVM). However, RVM is constructed under Bayesian framework and has probabilistic outputs correspondingly. Exponential model as (11) was utilized to fit the aging curves so as to estimate the model parameters $\alpha$ and $\beta$.

$$
R_{n}=\alpha \exp (-\beta t)
$$

The estimated parameters are used to form an initial state vector of a life prediction. Thus, EIS technique can provide quantified internal parameters to fit a regressive curve more accurately.

\section{B. State Estimation}

As mentioned in Section III.B, coulomb counting can be used to estimate the battery capacity. However, due to coulomb efficiency, the loss of the charge needs to be compensated to estimate SOC accurately. At the same time, both the effects of self-discharge and self-recharge should be considered. $\mathrm{Ng}$. proposed an advanced coulomb counting method to estimate SOC and SOH [10]. Considering the weakness of coulomb counting, charge and discharge stages should be separated for SOC calculation. The core idea is to build up an individual correction coefficient to calibrate the DOD through monitoring charge-discharge $I_{b}$ with a 2 seconds monitoring frequency. Thereby, the accurate SOC is established and recalibrated in distinctive stage

$$
\begin{gathered}
S_{O C}(t)=100 \%-D_{O D}(t) \\
\Delta D_{O D}=\frac{-\int_{t_{0}}^{t_{0}+\tau} I_{b}(t) d t}{Q_{\text {rated }}} \times 100 \% \\
D_{O D}(t)=D_{O D}\left(t_{0}\right)+\eta \Delta D_{O D} \\
S_{O H} \Leftarrow S_{O C}-D_{O D}
\end{gathered}
$$


Where $\eta$ have different values at charge and discharge stage. Generally, this estimation method utilizes simple calculations and has an uncomplicated hardware requirement that can be easily implemented. Although $\mathrm{Ng}$. [10] also mentioned the estimation of $\mathrm{SOH}$, the weakness of the method is $\mathrm{SOH}$ cannot be estimated until the voltage reaches the cut-off voltage in each cycle. The determination of $\mathrm{SOH}$ is not a real-time result as a result of ignoring the process information in a fully charge-discharge cycle. Thus, the result of $\mathrm{SOH}$ cannot be considered comprehensively.

The contribution of EIS is to estimate SOC in electrode-electrolyte interface according to the impedance variations during a cell life. The linear correlation connected the $\mathrm{C} / 1$ (capacity at nominal rated current of $1 \mathrm{~A}$ ) capacity with the internal impedance parameter $\mathrm{R}_{\mathrm{E}}+\mathrm{R}_{\mathrm{CT}}$ [15], which reflected on electrochemical characteristics directly. Thus, internal impedance is a good indicator to quantify the loss of capacity. Although EIS provides a non-invasive method to extract PoF information from battery equivalent circuit for on-board battery monitoring, it is costly and requires bulky measurement equipment.

Kalman filter [16] is an intelligent for estimating the present value of the time-varying "state" of a dynamic linear system. However, the battery degradation is a typical nonlinear problem. The extended Kalman filter (EKF) [17] was developed with a linearization process at every time step to approximate the nonlinear system. A battery state-space model is as below:

$$
\begin{gathered}
x_{k+1}=f\left(x_{k}, u_{k}\right)+\omega_{k} ; \quad y_{k}=g\left(x_{k}, u_{k}\right)+v_{k} \\
\hat{x}_{k}^{-}=f\left(\hat{x}_{k-1}^{+}, u_{k-1}\right) \\
\sum_{\bar{x}, k}^{-}=A_{k-1} \sum_{\bar{x}, k-1}^{+} \hat{A}_{k-1}^{T}+\mid \sum_{\omega} \\
L_{k}=\sum_{\bar{x}, k}^{-} \hat{C}_{k}^{T}\left[\widehat{C}_{k} \sum_{\bar{x}_{, k}}^{-} \hat{C}_{k}^{T}+\sum_{v}\right]^{-1}
\end{gathered}
$$

Where $f$ denotes a nonlinear state transition function, and $g$ denotes a nonlinear measurement function. $x$ denotes the state, $y$ is the measured output. Thus, a predicted state and output can be computed from the previous estimation. (14), (15), and (16) compute state estimate time update, error covariance time update, and Kalman gain matrix [16]. $\omega_{k}$ and $v_{k}$ follow independent, zero-mean, Gaussian noise processes of covariance matrices $\sum_{\omega}$ and $\sum_{v}$ respectively. Gaussian noise cannot be eliminated in the iteration process. Thus, a desired probability density function (PDF) is approximated by a Gaussian. An estimation result is subject to the deviation from a true distribution and an initial estimation of the state. Thus, if the initial estimate of the state is off-target, the filter may diverge quickly.

\section{Life Prediction}

The analytical model stated in Equation (5) can be used to predict a time-to-failure of battery for a given load, which is viewed as the constant or variable current. Thus, the end of battery life is a specific value corresponding to different load conditions. This kind of prediction cannot provide a degradation trend or a real-time performance of battery during charge-discharge cycles. As Rakhmatov said, the aging parameters that will reflect on degradation performance of the battery are valuable to discuss.
In Thomas's paper [14], the lifetime prediction of lithium-ion cells was realized in terms of accelerated degradation testing. Based on the empirical relative resistance model stated as (8), the simulation data were used in battery state estimation at the $40^{\circ} \mathrm{C}, 47.5^{\circ} \mathrm{C}$, and $55^{\circ} \mathrm{C}$ respectively as shown in fig.3. If the degradation process is assumed as a memoryless process, the resistance model will be accurate to estimate SOC and SOH of a battery. However, currently, whether that the rate of future degradation depends on the environment condition have not been proved. Thus the predictive accuracy based on this model in dynamic conditions also can not be accepted without suspicion. Furthermore, lack of analyzing the failure causes and mechanisms, similarly, the model can merely present the degradation trend but not present the RUL according to the specific failure threshold.

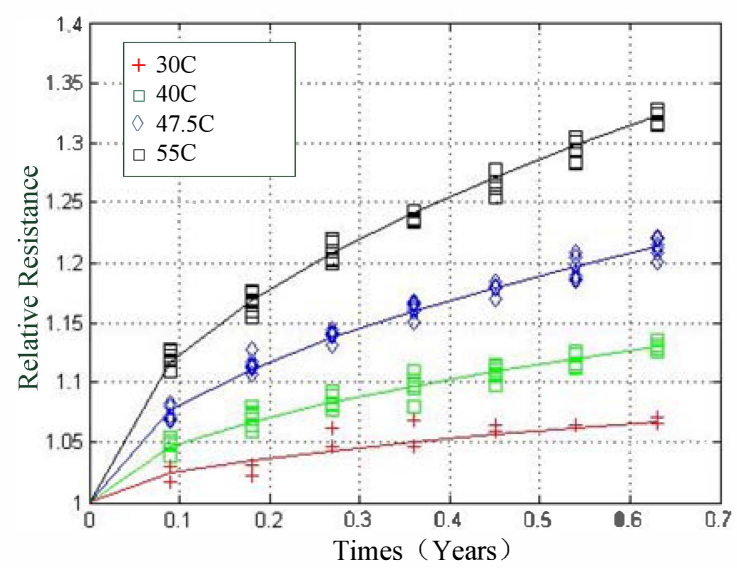

\section{Fig.3 Fitted Degradation Model [14]}

Autoregressive Moving Average (ARMA) is used to predict future values of time series data. It is a purely data-driven method and does not incorporate with any physics-based modeling during computation. The weakness is that this method will cause a large uncertainty margin to the long-term prediction. Additionally, it may not be possible to eliminate all non-stationarity from a dataset even though differencing repeatedly.

RVM combined with Particle Filter (PF) technology [15,19] was developed to estimate and predict the battery RUL. Particle Filter (PF) is a sequential Monte Carlo algorithm implementing with Bayesian filter. It utilizes a set of weighted samples or particles updated recursively to represent the PDF. The evolution in time of the system can be described Compared with Kalman Filter, PF makes few multivariate Gaussian assumptions. Thus, PF is more general and suitable to track a nonlinear process. Gobel [15] combined PF with RVM which provide not only a general rigorous construction for the dynamic state estimation problems, but also the estimates of RUL in PDF form. He presented that PF shows better accuracy when compared with other approaches above.

\section{Fusion TECHNiQue BASED ON PROGNOSTICS AND HEALTH MONITORING (PHM)}

By analyzing and comparing the characteristics of modeling, state estimation, and prognosis methods, we can see that a prediction of RUL of a battery allows exploiting the battery failure mechanism in conjunction with the data-driven technique.

PHM presents a new and comprehensive horizon to assess and optimize reliability of a battery. PHM can be used to estimate the battery failure with a high accuracy, and in turn, 
provide early alarm and improve the cost efficiency. Prognostic-based reliability [20] predicts a reliable situation in future by assessing the extent of deviation or the degradation of the battery under normal operating conditions. Health monitoring of batteries provides a way to respond in a preemptive manner and opportunistic manner to the anticipation of failures. Based on the prediction results, a recommendation for when should the battery be discarded, repaired or recharged could be made. These decisions can reduce the cost of replacement based on the extent of degradation.

PoF is an approach that utilizes the knowledge of a product's life cycle loading and failure mechanisms to assess reliability of the product $[20,21]$. For batteries, the external configuration, inner structure, and material variations can be characterized by a PoF approach. Through the accelerated life testing, the reliability of a battery is determined in terms of time-to-failure for a specific failure mechanism under a given load condition. The accelerated factor is quantified in PoF approach by comparing test loads with the environment loads. It will be used to determine the equivalent life if a battery runs at normal operating conditions.

As mentioned before, EIS is a non-invasive method to monitor the shifts in battery orientation or separation of materials. Besides, X-ray analysis, and CT scans are also good non-invasive methods to spot cracks, internal shorts, and delaminating of the electrodes from the current collectors. However, from the inherent failure mechanisms perspective, destructive failure analysis is more direct to observe the failure. Both the electrodes and the electrolyte are the internal attributes, such as the electron motion in electrolyte, and the volume expansion with gas evolution, which have a function on nonlinear degradation. All of these electrochemical characteristics can be conducted to build up a generalized degradation model or modify the existing models based on PoF of lithium-ion batteries.

Most current prediction technologies are still data-driven that monitor and record the health information of large multivariate systems through an online mechanism. The approaches are capable to detect intelligently and assess correlated trends in the system dynamically to estimate the current and future health. The data features, such as correlation, covariance and residuals etc., provide fault or damage information over time. With the aid of prognostic algorithms, it is possible to state from the beginning of battery degradation towards unhealthy occurrence. Comparing healthy states with unhealthy ones, it is possible to estimate the RUL or the time to failure of the battery.

This prediction method is based on fairly predictable degradation trend. We have known that the battery performance is subject to various external parameters, which will present non-exact, non-linear, and non-stationary characteristics due to the imperceptible electrochemical process. Depending on the data-driven technique, it will predict remaining useful life accurately near the failure point. If this is the case, the prediction makes no sense and can even lead to a diagnosis problem. Furthermore, if multivariable lead the battery degradation, data-driven cannot distinguish the corresponding relationship between the data features and the specific failure causes.

Our objective is to develop a formal and generalized way to predict the RUL of a battery with high accuracy and high efficiency, which leads to save the qualification testing time. The proposed fusion approach combines the merits of both PoF and data-driven techniques. It not only extracts the root causes and failure mechanisms that contribute to product failure, but also addresses the complexity and the density of systems by utilizing operational data. Simply, it can improve the prediction accuracy (a smaller deviation of failure time) and precision (a narrower confidence bound). The method is worth to extending and applying into prognostics for battery qualification and health monitoring. The framework is given in fig.4.

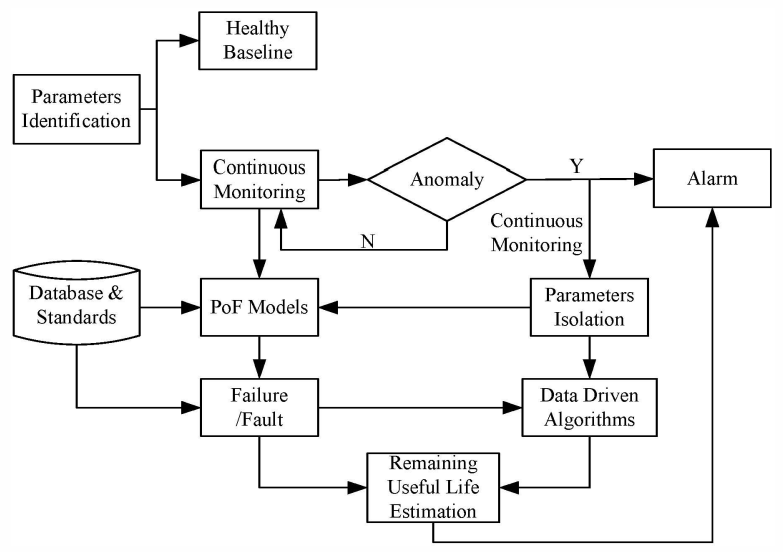

\section{Fig.4 HeAlth MONITORING ON LithiUM-ION BATTERY}

Before the fusion work, the battery degradation under common operating conditions is used to achieve the baseline data and to select the fundamental monitored parameters. Through monitoring battery status continuously, some parameters that contribute significantly to the observed anomaly are isolated and used to choose PoF model. For example, as mentioned before, the higher the discharge rate, the lower the battery capacity. $\mathrm{Ci}$ [22] also gave a relationship between the available capacity and the specified discharge rate with nonlinear characteristics. When a discharge rate goes up to the threshold, a usable capacity will drop to $80 \%$ of its original value to failure. Hence, the C-rate becomes a primary parameter relevant to battery degradation. On one hand, the discharge rate should be extracted and input into a PoF model to conduct a single factor experiment. We can conduct the accelerated life test with the different factor levels and predict the RUL at the normal operating condition in terms of the certain failure mechanism. On the other hand, the threshold of C-rate will also become an alarm signal from safety concerns. (Other specified parameters will not be introduced here.) Furthermore, depending on the different specific failure causes and thresholds, the data-driven algorithms can classify correlated mechanisms that affect the battery degradation. Thus, the RUL of battery can present a more accurate and precise result. This kind of monitoring and predictive method will have a great promotion on battery qualification with time-saving.

\section{CONCLUSIONS}

Prognostics and health monitoring is an enabling technique to conduct the state estimation and predict the remaining useful life of battery at an early stage of detection. To solve the widely concerned issues of battery health, this paper discussed the common failure mechanisms of the battery and compared the existing methods of battery modeling, state estimation and life prediction under health monitoring. Through combining the inherent failure characteristics of the Lithium-ion battery with the existing research methods, we proposed to apply a fusion technique combining health monitoring and the prognostics of battery life. The specific method was presented as a 
comparative study that makes a solid foundation on the further study.

\section{ACKNOWLEDGMENT}

The work described in this paper was fully supported by a grant from the Research Grants Council of the Hong Kong Special Administrative Region, China (CityU8/CRF/09).

\section{REFERENCES}

[1] http://www.dellbatteryprogram.com/

[2] http://bpr.hpordercenter.com/hbpr/

[3] Daniel C.Cox, Regina Perez-Kite, "Battery State of Health Monitoring, Combine Conductance Technology With Other Measurement Parameters for Real-Time Battery Performance Analysis", Twenty-second Internatonal Telecommunications Energy Conference, September 2000, pp. 342-347.

[4] Kozlowski,J.D., "Electrochemical Cell Prognostics using Online Impedance Measurements and Model-Based Data Fusion Techniques", Aerospace Conference, 2003.Proceedings. 2003 IEEE, vol. 7, pp. 3257-3270.

[5] Behnood Gholami, Wassim M.Haddad, Allen R.Tannenbaum, "Relevance Vector Machine Learning for Neonate Pain Intensity Assessment Using Digital Imaging", IEEE Transactions on biomedical engineering, vol. 57, No. 6, June 2010, pp. 1457-1466.

[6] http://products.panasonic-industrial.com/datasheets/en/CGR18650C.pdf

[7] T.Guena, P.Leblanc, "How depth of discharge affects the cycle life of Lithium-Methal-Polymer Batteries", Telecommunications Energy Conference, 2006, pp. 1-8.

[8] http://www.che.sc.edu/faculty/popov/drbnp/WebSite/MSA-calendar.pdf.

[9] Ira Bloom, Jon Christophersen, Kevin Gering,"Differential voltage analyses of high-power lithium-ion cells: 2. Applications", Journal of Power Sources, vol. 139, Issues 1-2, Janurary 2005, pp. 304-313.

[10] Kong Soon Ng, Chin-Sien Moo, Yi-Ping Chen, Yao-Ching Hsieh, "Enhanced coulomb method for estimating state-of-charge and state-of-health of lithium-ion batteries", Applied energy, vol. 86, Issue 9, September 2009, pp. 1506-1511.

[11] Holger Blanke, Oliver Bohlen, Stephan Buller, etc. "Impedance measurements on lead-acid batteries for state-of-charge, state-of-health and cranking capability prognosis in electric and hybrid electric vehicles", Journal of Power Sources, vol. 144, Issue 2, June 2005, pp. 418-425.

[12] Daler Rahkmatov, Sarma Vrudhula, and Deborah a.Wallach, "A model for battery lifetime analysis for organizing applications on a pocket computer", IEEE Transactions on very large scale integration (VLSI) Systems, vol.11, No.6, Decemeber 2003, pp. 1019-1030

[13] D.Linden, Handbook of Batteries. New York: McGraw-Hill, 1995.

[14] E.V.Thomas, I.Bloom, J.P.Christophersen, V.S.Battaglia, "Statistical methodology for predicting the life of lithium-ion cells via accelerated degradation testing", Journal of Power Source, vol. 184, Issue 1, September 2008, pp. 312-317.

[15] Kai Goebel, Bhaskar Saha, Abhinav Saxena, Jose R. Celaya, and Jon P. Chirtophersen,"Prognostics in Health Management", Instrumentation \& Measurement Magazine, IEEE, vol. 11, Issue 4, pp. 33-40.

[16] Gregoy L.Plett, "Extended Kalman filtering for battery management systems of LiPB-based HEV battery packs Part 1. Background", Journal of Power Sources, vol. 134, Issue 2, August 2004, pp. 252-261.

[17] Gregoy L.Plett, "Extended Kalman filtering for battery management systems of LiPB-based HEV battery packs Part 2. Modeling and identification", Journal of Power Sources, vol. 134, Issue 2, August 2004 pp. 262-276

[18] Behnood Gholami, Wassim M.Haddad, Allen R.Tannenbaum, "Relevance Vector Machine Learning for Neonate Pain Intensity Assessment Using Digital Imaging", IEEE Transactions on biomedical engineering, vol. 57, No. 6, June 2010, pp. 1457-1466.

[19] Saha, B, Goebel, K, Poll, S, Christophersen, J., "An integrated approach to battery health monitoring using Bayesian regression and state estimation", Autotestcon, IEEE, 2007, pp. 646-653.

[20] Michael G. Pecht, Prognostics and Health Management of Electronics, Wiley-Interscience, New Jersy, NJ, 2008.
[21] Michael Peht, and Jie Gu, "Physics-of-failure-based prognostics for electronic products", Transactions of the Institute of Measurement and Control, vol. 31, No. 3-4, June/August 2009, pp. 309-322.

[22] Song Ci et al., "A Novel Design of Adaptive Reconfigurable Multicell Batery for Power-Aware Embedded Networked Sensing Systems", Global Telecommunications Conference, 2007, IEEE, pp. 1043-1047. 